\title{
Edgewise Cohen-Macaulay connectivity of partially ordered sets
}

\author{
Christos A. Athanasiadis \\ Department of Mathematics \\ University of Athens \\ Panepistimioupolis \\ Athens 15784, Hellas (Greece) \\ caath@math.uoa.gr
}

\author{
Myrto Kallipoliti \\ Department of Mathematics \\ University of Vienna \\ Oskar-Morgenstern-Platz 1 \\ 1090 Vienna, Austria \\ myrto.kallipoliti@univie.ac.at
}

November 6, 2015

\begin{abstract}
The proper parts of face lattices of convex polytopes are shown to satisfy a strong form of the Cohen-Macaulay property, namely that removing from their Hasse diagram all edges in any closed interval results in a Cohen-Macaulay poset of the same rank. A corresponding notion of edgewise Cohen-Macaulay connectivity for partially ordered sets is investigated. Examples and open questions are discussed.
\end{abstract}

Keywords: Partially ordered set, order complex, Cohen-Macaulay complex, Cohen-Macaulay connectivity, Gorenstein* lattice.

\section{Introduction and results}

Cohen-Macaulay simplicial complexes and partially ordered sets (posets) have been studied intensely for the past few decades, mainly due to their importance in several areas of mathematics; see [6] for an informal discussion and references.

Cohen-Macaulay connectivity, introduced by Baclawski [3], is a sophisticated notion of connectivity for simplicial complexes and posets; it includes as a special case the notion of vertexconnectivity of graphs. This paper investigates a new notion of connectivity for posets which is motivated by that of edge-connectivity of graphs. Given a positive integer $k$, a poset $\mathcal{P}$ is called edgewise $k$-Cohen-Macaulay if $\mathcal{P}$ is Cohen-Macaulay of rank at least $k-1$ and the removal from the Hasse diagram of $\mathcal{P}$ of all edges in any closed interval of $\mathcal{P}$ of rank less than $k$ results in a Cohen-Macaulay poset of the same rank as $\mathcal{P}$. The main problem we propose to study asks to determine the largest integer $k$ for which a given poset is edgewise $k$-Cohen-Macaulay; this integer is called the edgewise Cohen-Macaulay connectivity.

The main result of this paper solves this problem for one important class of posets, namely the proper parts of Gorenstein* lattices. We call edgewise strongly Cohen-Macaulay the posets whose edgewise Cohen-Macaulay connectivity is maximum possible, equal to one more than their rank. Thus, edgewise strongly Cohen-Macaulay posets have the property that removing from their Hasse diagram all edges in any closed interval results in a Cohen-Macaulay poset of 
the same rank. Recall that the class of Gorenstein* lattices includes all face lattices of convex polytopes and that the proper part $\overline{\mathcal{L}}$ of a lattice $\mathcal{L}$ is the poset obtained from $\mathcal{L}$ by removing its minimum and maximum elements.

Theorem 1.1 The poset $\overline{\mathcal{L}}$ is edgewise strongly Cohen-Macaulay for every Gorenstein* lattice $\mathcal{L}$.

This theorem is proven in Section 4 using methods of topological combinatorics. The notion of edgewise Cohen-Macaulay connectivity, adopted in this paper, is introduced in Section 3 , where elementary properties of edgewise $k$-Cohen-Macaulay posets are established, examples are listed and a comparison to $k$-Cohen-Macaulay posets, in the sense of Baclawski [3, is given for $k=2$ (Proposition 3.4). Basic definitions and notation needed to understand this paper are summarized in Section 2. Some open problems and related remarks are discussed in Section 5 ,

\section{Preliminaries}

This section fixes notation and recalls basic definitions and background on the combinatorics and topology of simplicial complexes and partially ordered sets. For more information on these topics and any undefined terms, the reader is referred to the sources [5] [11] [12, Chapter 3] [13. Basic background on algebraic topology can be found in $[10$.

Simplicial complexes. An (abstract) simplicial complex on a ground set $E$ is a collection $\Delta$ of subsets of $E$, called faces, such that $F \subseteq G \in \Delta$ implies $F \in \Delta$. The elements $v \in E$ for which $\{v\} \in \Delta$ are the vertices of $\Delta$. The dimension of a face $F \in \Delta$ is defined as one less than the cardinality of $F$. The dimension of $\Delta$, denoted $\operatorname{dim}(\Delta)$, is the maximum dimension of its faces. This paper is concerned with order complexes of finite posets (discussed later in this section); in particular, all simplicial complexes considered here will be finite.

Every simplicial complex $\Delta$ has a geometric realization [5, Section 9], uniquely defined up to homeomorphism; it will be denoted by $|\Delta|$. All topological properties of $\Delta$ mentioned in the sequel refer to those of $|\Delta|$.

A subcollection $\Gamma \subseteq \Delta$ which is a simplicial complex on its own is called a subcomplex. The geometric realization $|\Gamma|$ of such a complex may be considered as a subspace of $|\Delta|$. The link of a face $F \in \Delta$ is the subcomplex defined as $\operatorname{link}_{\Delta}(F)=\{G \backslash F: F \subseteq G \in \Delta\}$. The open star of a face $F \in \Delta$ is defined as $\operatorname{st}_{\Delta}(F)=\{G \in \Delta: F \subseteq G\}$. Its complement in $\Delta$, denoted $\operatorname{cost}_{\Delta}(F)$, is a subcomplex of $\Delta$ called the contrastar of $F$; it consists of all faces of $\Delta$ which do not contain $F$. A subcomplex $\Gamma$ of $\Delta$ is said to be vertex-induced if $\Gamma$ contains all faces $F \in \Delta$ for which all elements of $F$ are vertices of $\Gamma$. Given $A \subseteq E$, the (induced) subcomplex consisting of all faces of $\Delta$ which are disjoint from $A$ will be denoted by $\Delta \backslash A$. The complex $\Delta$ is said to be a cone with apex $v \in E$ if $F \cup\{v\} \in \Delta$ for every $F \in \Delta$; such a complex is contractible.

The following standard lemma will be used in Section 4 .

Lemma 2.1 ([10, Lemma 70.1]) Let $\Delta$ be a simplicial complex and $\Gamma$ be a vertex-induced subcomplex with vertex set $A$. Then the space $|\Delta \backslash A|$ is a deformation retract of $|\Delta| \backslash|\Gamma|$.

A simplicial complex $\Delta$ is Cohen-Macaulay over a field $\mathbf{k}$ if $\widetilde{H}_{i}\left(\operatorname{link}_{\Delta}(F), \mathbf{k}\right)=0$ for all $F \in \Delta$ (including the empty face) and $i<\operatorname{dim}\left(\operatorname{link}_{\Delta}(F)\right)$, where $\widetilde{H}_{*}(\Gamma, \mathbf{k})$ denotes reduced simplicial homology of $\Gamma$ with coefficients in $\mathbf{k}$, and Gorenstein* over $\mathbf{k}$ if it is Cohen-Macaulay 

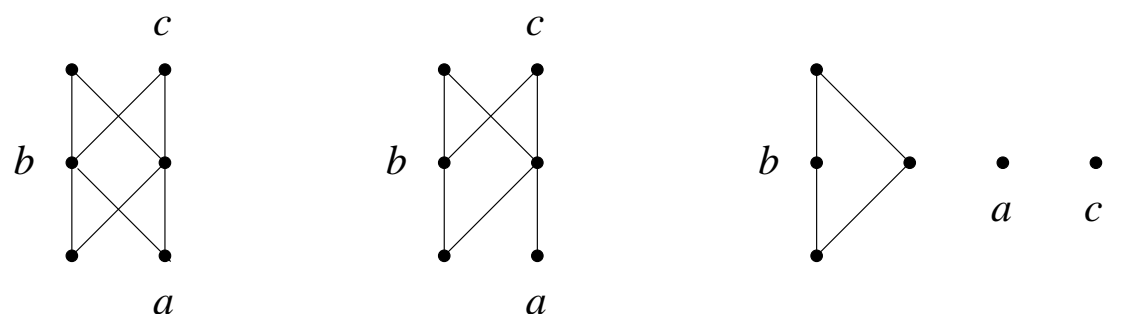

Figure 1: A poset $\mathcal{P}$ and the posets $\mathcal{P} \ominus[a, b]$ and $\mathcal{P} \ominus[a, c]$

over $\mathbf{k}$ and $\widetilde{H}_{i}\left(\operatorname{link}_{\Delta}(F), \mathbf{k}\right)=\mathbf{k}$ for all $F \in \Delta$ and $i=\operatorname{dim}\left(\operatorname{link}_{\Delta}(F)\right)$. Given a positive integer $k$, the complex $\Delta$ is $k$-Cohen-Macaulay (or doubly Cohen-Macaulay, for $k=2$ ) over $\mathbf{k}$ if $\Delta \backslash A$ is Cohen-Macaulay over $\mathbf{k}$ of the same dimension as $\Delta$ for every subset $A$ of the ground set of cardinality less than $k$ (including $A=\varnothing$ ).

Every Cohen-Macaulay simplicial complex $\Delta$ is pure, meaning that all maximal (with respect to inclusion) faces of $\Delta$ have dimension equal to $\operatorname{dim}(\Delta)$. The properties of being CohenMacaulay, Gorenstein* or doubly Cohen-Macaulay are all topological, i.e., they depend only on the homeomorphism type of $|\Delta|$ and the field $\mathbf{k}$; more precise statements appear in [11, 15]. From now on, we consider the field $\mathbf{k}$ fixed and suppress it from our terminology. Our results continue to hold if $\mathbf{k}$ is replaced by the ring of integers.

Partially ordered sets. This paper considers only finite partially ordered sets (posets). Thus, a poset is a pair $\mathcal{P}=(P, \leq)$ consisting of a finite set $P$ and a partial order (reflexive, antisymmetric and transitive binary relation $) \leq$ on $P$. We will denote by $\mathcal{E}(\mathcal{P})$ the set of all cover relations of $\mathcal{P}$, meaning ordered pairs $(a, b) \in P \times P$ with $a<b$ for which no $x \in P$ satisfies $a<x<b$. The Hasse diagram of $\mathcal{P}$ is the directed graph with vertex set $P$ and edge set $\mathcal{E}(\mathcal{P})$. We assume familiarity with drawing posets via their Hasse diagrams; see Figure 1 on the left for an example of a poset with six elements and eight cover relations.

Given elements $a, b \in P$ with $a \leq b$, the closed interval $[a, b]_{\mathcal{P}}$ is defined as the (induced) subposet of $\mathcal{P}$ on the set $\{x \in P: a \leq x \leq b\}$, where the subscript $\mathcal{P}$ can be dropped if there is no danger of confusion. Similar remarks apply to open and half-open intervals. The subposets on the sets $\{x \in P: x \geq a\}$ and $\{x \in P: x \leq b\}$ will be denoted by $\mathcal{P}_{\geq a}$ and $\mathcal{P}_{\leq b}$, respectively. The set of two-element closed intervals (in other words, the set of undirected edges of the Hasse diagram) of $\mathcal{P}$ will be denoted by $E(\mathcal{P})$.

A chain (respectively, antichain) in $\mathcal{P}$ is any subset of $P$ which consists of pairwise comparable (respectively, incomparable) elements. The poset $\mathcal{P}$ is graded if all maximal (with respect to inclusion) chains in $\mathcal{P}$ have the same length (defined as one less than their cardinality). This common length is then called the rank of $\mathcal{P}$ and denoted by $\operatorname{rank}(P)$.

The poset $\mathcal{P}$ is bounded if it has a minimum element $\hat{0}$ and a maximum element $\hat{1}$; the elements of $P$ covering $\hat{0}$ are called atoms and those covered by $\hat{1}$ are called coatoms. The proper part of a bounded poset $\mathcal{P}$ is defined as the subposet $\overline{\mathcal{P}}$ obtained from $\mathcal{P}$ by removing $\hat{0}$ and $\hat{1}$. Conversely, the poset obtained from a poset $\mathcal{Q}$ by artificially adding a minimum element $\hat{0}$ and a maximum element $\hat{1}$ is denoted by $\widehat{\mathcal{Q}}$. The ordinal sum of two posets $\left(P, \leq_{P}\right)$ and $\left(Q, \leq_{Q}\right)$ on disjoint ground sets $P$ and $Q$ is the set $P \cup Q$ partially ordered by setting $x \leq y$ if $x, y \in P$ and $x \leq_{P} y$, or $x, y \in Q$ and $x \leq_{Q} y$, or $x \in P$ and $y \in Q$, for $x, y \in P \cup Q$.

A lattice is a poset any two elements $x, y$ of which have a least upper bound, called the join and denoted by $x \vee y$, and a greatest lower bound, called the meet and denoted by $x \wedge y$. Cleary, 
every finite lattice is bounded. Such a lattice $\mathcal{L}=(L, \leq)$ is called atomic if every element of $L$ is the join of atoms, relatively atomic if every closed interval of $\mathcal{L}$ is atomic and semimodular if for all $x, y \in L$, the join $x \vee y$ covers $y$ whenever $x$ covers the meet $x \wedge y$. A lattice which is both atomic and semimodular is called geometric.

The order complex associated to a poset $\mathcal{P}=(P, \leq)$ is the simplicial complex on the ground set $P$ whose faces are the chains of $\mathcal{P}$; it is denoted $\Delta(\mathcal{P})$. Note that $\mathcal{P}$ is graded if and only if $\Delta(\mathcal{P})$ is pure; in that case, $\operatorname{rank}(\mathcal{P})=\operatorname{dim}(\Delta(\mathcal{P}))$. The poset $\mathcal{P}$ is called Cohen-Macaulay, Gorenstein* or $k$-Cohen-Macaulay if $\Delta(\mathcal{P})$ has the corresponding property. An equivalent definition for $\mathcal{P}$ to be Cohen-Macaulay is that $\widetilde{H}_{i}(\Delta(\mathcal{J}), \mathbf{k})=0$ for all open intervals $\mathcal{J}$ in $\widehat{\mathcal{P}}$ and indices $i<\operatorname{rank}(\mathcal{J})$. A lattice $\mathcal{L}$ is said to be Gorenstein* (by slight abuse of language) if its proper part $\overline{\mathcal{L}}$ is a Gorenstein* poset. The class of Gorenstein* lattices includes all face lattices of convex polytopes and, more generally, regular cell decompositions of the sphere having the intersection property.

\section{Edgewise Cohen-Macaulay connectivity}

This section introduces the notion of edgewise Cohen-Macaulay connectivity for posets, establishes some of its elementary properties and compares it with the standard notion of CohenMacaulay connectivity, due to Baclawski [3].

Given a poset $\mathcal{P}=(P, \leq)$ and a closed interval $\mathcal{I} \subseteq \mathcal{P}$, we denote by $\mathcal{P} \ominus \mathcal{I}$ the poset whose Hasse diagram is obtained from that of $\mathcal{P}$ by removing all edges within $\mathcal{I}$. Equivalently, $\mathcal{P} \ominus \mathcal{I}$ is the unique poset on the ground set $P$ which satisfies $\mathcal{E}(\mathcal{P} \ominus \mathcal{I})=\mathcal{E}(\mathcal{P}) \backslash \mathcal{E}(\mathcal{I})$. An example is shown in Figure 1, Note that the elements of $\mathcal{I}$ are pairwise incomparable in $\mathcal{P} \ominus \mathcal{I}$.

The main definition of this paper is as follows.

Definition 3.1 Let $k$ be a positive integer. A Cohen-Macaulay poset $\mathcal{P}$ is called

(a) edgewise $k$-Cohen-Macaulay if $\operatorname{rank}(P) \geq k-1$ and $\mathcal{P} \ominus \mathcal{I}$ is Cohen-Macaulay of the same rank as $\mathcal{P}$ for every closed interval $\mathcal{I} \subseteq \mathcal{P}$ of rank less than $k$; and

(b) edgewise strongly Cohen-Macaulay if $\mathcal{P} \ominus \mathcal{I}$ is Cohen-Macaulay of the same rank as $\mathcal{P}$ for every closed interval $\mathcal{I} \subseteq \mathcal{P}$.

The largest integer $k$ for which $\mathcal{P}$ is edgewise $k$-Cohen-Macaulay is called the edgewise CohenMacaulay connectivity of $\mathcal{P}$.

In particular, the edgewise 1-Cohen-Macaulay posets are exactly the Cohen-Macaulay posets, whereas a poset $\mathcal{P}$ is edgewise 2-Cohen-Macaulay (or edgewise doubly Cohen-Macaulay) if it is Cohen-Macaulay of positive rank and $\mathcal{P} \ominus e$ is Cohen-Macaulay of the same rank as $\mathcal{P}$ for every $e \in E(\mathcal{P})$.

Example 3.2 (a) A poset of rank one is Cohen-Macaulay (respectively, 2-Cohen-Macaulay or edgewise 2-Cohen-Macaulay) if and only if it is connected (respectively, 2-vertex-connected or 2-edge-connected) as a graph.

(b) Chains are Cohen-Macaulay but neither 2-Cohen-Macaulay, nor edgewise 2-CohenMacaulay; their edgewise Cohen-Macaulay connectivity is equal to one.

(c) The poset on the left of Figure 1 is 2-Cohen-Macaulay and edgewise 2-Cohen-Macaulay but neither 3-Cohen-Macaulay, nor edgewise 3-Cohen-Macaulay. Thus, its edgewise CohenMacaulay connectivity is equal to two; see Remark 3.8 for a generalization. 
(d) Let $\mathcal{B}_{n}$ be the Boolean lattice of rank $n$ [12, Example 3.1.1], consisting of all subsets of $\{1,2, \ldots, n\}$, ordered by inclusion. It follows from Theorem 1.1 that $\overline{\mathcal{B}}_{n}$ is edgewise strongly Cohen-Macaulay for every $n$.

The following elementary properties of edgewise $k$-Cohen-Macaulay posets are similar to corresponding properties of $k$-Cohen-Macaulay posets.

Proposition 3.3 (a) The class of edgewise $(k+1)$-Cohen-Macaulay posets is strictly included in that of edgewise $k$-Cohen-Macaulay posets for all $k \geq 1$.

(b) Every poset which is edgewise $k$-Cohen-Macaulay for some $k \geq 2$ contains at least two minimal and at least two maximal elements.

(c) Let $\mathcal{P}$ be edgewise $k$-Cohen-Macaulay. Then every open interval $\mathcal{J}$ in $\widehat{\mathcal{P}}$ is edgewise $r$ Cohen-Macaulay for $r=\min \{k, \operatorname{rank}(\mathcal{J})+1\}$.

Proof. Part (a) is a direct consequence of Definition 3.1. The inclusion is strict since, by the same definition, edgewise $k$-Cohen-Macaulay posets of rank $k-1$ are not edgewise $(k+1)$ Cohen-Macaulay.

To verify part (b), suppose $\mathcal{P}$ is Cohen-Macaulay with a unique minimal (hence minimum) element $\hat{0}$, or a unique maximal (hence maximum) element $\hat{1}$ and note that removing any edge which contains $\hat{0}$ or $\hat{1}$ from the Hasse diagram of $\mathcal{P}$ results in a poset which is either not graded, or which has rank less than that of $\mathcal{P}$. Thus, such a poset is not edgewise doubly Cohen-Macaulay and hence not edgewise $k$-Cohen-Macaulay for any $k \geq 2$ either.

To prove part (c) note that $\mathcal{J}$ is Cohen-Macaulay, as an interval in a Cohen-Macaulay poset, and consider any closed interval $\mathcal{I}$ of rank less than $k$ in $\mathcal{J}$. Then $\mathcal{I}$ is a closed interval of rank less than $k$ in $\mathcal{P}$ as well and thus $\mathcal{P} \ominus \mathcal{I}$ is Cohen-Macaulay of the same rank as $\mathcal{P}$. As a result, $\widehat{\mathcal{P}} \ominus \mathcal{I}$ is Cohen-Macaulay of the same rank as $\widehat{\mathcal{P}}$. Being an open interval in $\widehat{\mathcal{P}} \ominus \mathcal{I}$, the poset $\mathcal{J} \ominus \mathcal{I}$ must be Cohen-Macaulay of the same rank as $\mathcal{J}$ and the proof follows.

The next statement exhibits a large class of edgewise doubly Cohen-Macaulay posets; it implies, for instance, that $\mathcal{P}$ is edgewise doubly Cohen-Macaulay whenever $|\Delta(\mathcal{P})|$ is homeomorphic to a sphere of positive dimension.

Proposition 3.4 Every doubly Cohen-Macaulay poset of positive rank is edgewise doubly CohenMacaulay.

To prepare for the proof of Proposition 3.4, we make the following observation. Recall from Section 2 that $\operatorname{cost}_{\Delta(\mathcal{P})}(F)$ is the simplicial complex of chains in a poset $\mathcal{P}$ which do not contain a given chain $F \in \Delta(\mathcal{P})$. Since no chain in $\mathcal{P} \ominus[a, b]$ can contain two distinct elements of $[a, b]$, we have

$$
\Delta(\mathcal{P} \ominus[a, b]) \subseteq \bigcap_{a \leq x<y \leq b} \operatorname{cost}_{\Delta(\mathcal{P})}(\{x, y\})
$$

for every closed interval $[a, b] \subseteq \mathcal{P}$.

Lemma 3.5 Let $\mathcal{P}$ be a finite poset which contains no three-element closed interval. Then $\Delta(\mathcal{P} \ominus e)=\operatorname{cost}_{\Delta(\mathcal{P})}(e)$ for every $e \in E(\mathcal{P})$. 

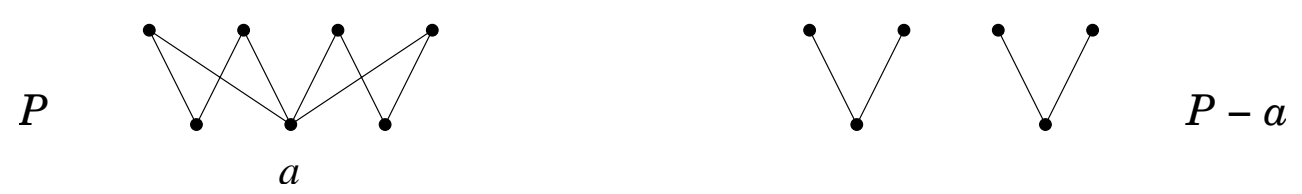

Figure 2: An edgewise 2-Cohen-Macaulay poset $\mathcal{P}$ which is not 2-Cohen-Macaulay

Proof. Let $e \in E(\mathcal{P})$. We claim that for every pair $\{u, v\}$ of elements of $\mathcal{P}$ other than $e$ with $u<v$, there exists a saturated chain in $\mathcal{P}$ with minimum element $u$ and maximum element $v$ which does not contain $e$. Indeed, this is trivial if $\{u, v\} \in E(\mathcal{P})$. Otherwise, one can consider any maximal chain of the closed interval $[u, v]$ of $\mathcal{P}$ and in case this chain contains both elements of $e$, since $\mathcal{P}$ contains no three-element closed intervals, one can replace one of the elements of $e$ with other elements of $\mathcal{P}$ to obtain a chain with the desired properties.

As a result of the claim, if $u<v$ holds in $\mathcal{P}$ for some pair $(u, v)$ of elements of $\mathcal{P}$ other than $e$, then $u<v$ holds in $\mathcal{P} \ominus e$ as well. This implies that every chain in $\mathcal{P}$ which does not contain $e$ is also a chain in $\mathcal{P} \ominus e$, in other words that $\operatorname{cost}_{\Delta(\mathcal{P})}(e) \subseteq \Delta(\mathcal{P} \ominus e)$. The reverse inclusion is a special case of (1).

Proof of Proposition 3.4. Let $\mathcal{P}$ be a doubly Cohen-Macaulay poset and let $e \in E(\mathcal{P})$. We need to show that $\Delta(\mathcal{P} \ominus e)$ is Cohen-Macaulay of the same dimension as $\Delta(\mathcal{P})$. Since the class of doubly Cohen-Macaulay complexes is closed under taking links of faces, no open interval in $\mathcal{P}$ can be a singleton. Thus, from Lemma 3.5 we get $\Delta(\mathcal{P} \ominus e)=\operatorname{cost}_{\Delta(\mathcal{P})}(e)$. Moreover, since $\Delta(\mathcal{P})$ is doubly Cohen-Macaulay, [9, Proposition 2.8] and its proof imply that $\operatorname{cost}_{\Delta(\mathcal{P})}(e)$ is Cohen-Macaulay of the same dimension as $\Delta(\mathcal{P})$ and the proof follows.

We conclude this section with the following remarks.

Remark 3.6 (a) The converse of Proposition 3.4 is false. Figure 2 shows an edgewise 2-CohenMacaulay poset $\mathcal{P}$ of rank one which is not 2-Cohen-Macaulay. For an example of rank two, consider the ordinal sum $\mathcal{Q}$ of $\mathcal{P}$ with a two-element antichain, shown on the left of Figure 3 , One can easily check that $\mathcal{Q} \ominus e$ is pure-shellable of rank two for every $e \in E(\mathcal{Q})$ and conclude that $\mathcal{Q}$ is edgewise 2-Cohen-Macaulay. On the other hand, $\mathcal{Q} \backslash a$ is not Cohen-Macaulay since, for instance, removing its two maximal elements results in a rank-selected subposet which is disconnected of rank one and hence not Cohen-Macaulay. Therefore, $\mathcal{Q}$ is not 2-CohenMacaulay.

We leave it to the interested reader to check that, more generally, the ordinal sum of $\mathcal{P}$ and any number of two-element antichains, taken in this order, is an edgewise 2-Cohen-Macaulay poset which is not 2-Cohen-Macaulay.

(b) The converse of Proposition 3.4 does hold for the class of semimodular lattices. For a semimodular lattice $\mathcal{L}$, the following conditions are in fact equivalent: (i) $\mathcal{L}$ is geometric; (ii) $\overline{\mathcal{L}}$ is 2-Cohen-Macaulay; and (iii) $\overline{\mathcal{L}}$ is edgewise 2-Cohen-Macaulay. The equivalence (i) $\Leftrightarrow$ (ii) is the content of [3, Theorem 3.1]. Suppose that $\mathcal{L}$ is semimodular but not geometric. Then, as discussed in the proof of [3, Theorem 3.1], there exists an element $b \in \mathcal{L}$ which is not an atom and covers a unique element $a$. As a result, either $\overline{\mathcal{L}}$ has a unique maximal element (equal to $a$, if $b=\hat{1}$ ), or else $\mathcal{L}$ has an open interval of rank one which contains $a$ and $b$ and hence is not edgewise 2-Cohen-Macaulay. Proposition 3.3 implies that $\overline{\mathcal{L}}$ is not edgewise 2-Cohen-Macaulay either. We have shown that (iii) $\Rightarrow$ (i). The implication (ii) $\Rightarrow$ (iii) follows from Proposition 3.4 

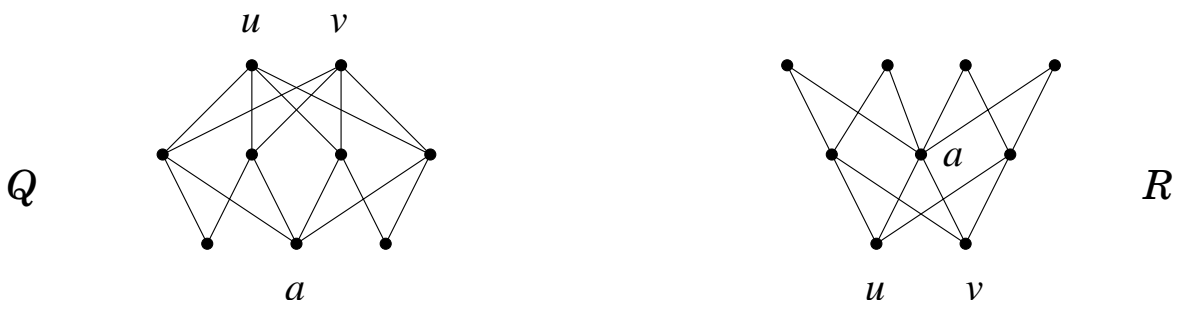

Figure 3: Two homeomorphic posets

Remark 3.7 (a) Contrary to the situation with (double) Cohen-Macaulayness, edgewise double Cohen-Macaulayness is not a topological property. Consider, for instance, the posets $\mathcal{Q}$ and $\mathcal{R}$ shown in Figure 3, Both order complexes $\Delta(\mathcal{Q})$ and $\Delta(\mathcal{R})$ are homeomorphic to the suspension over the wedge of two circles. However, $\mathcal{Q}$ is edgewise 2-Cohen-Macaulay, as already commented in Remark 3.6 (a), and $\mathcal{R}$ is not, since the poset $(\mathcal{R} \ominus[u, a])_{>u}$ is disconnected of rank one and hence $\mathcal{R} \ominus[u, a]$ is not Cohen-Macaulay.

Consideration of the ordinal sum of $n-1$ two-element antichains and of the proper part $\overline{\mathcal{B}}_{n}$ of the Boolean lattice of rank $n$ shows that edgewise $k$-Cohen-Macaulayness for $k \geq 3$ and edgewise strong Cohen-Macaulayness are not topological properties either.

(b) The ordinal sum of edgewise doubly Cohen-Macaulay (respectively, edgewise strongly Cohen-Macaulay) posets is not always edgewise doubly Cohen-Macaulay (respectively, edgewise strongly Cohen-Macaulay). For a counterexample, take the ordinal sum of two two-element antichains and the poset of Figure 2 and argue as with the poset $\mathcal{R}$ of part (a).

Remark 3.8 The natural generalizion of Proposition 3.4 to $k$-Cohen-Macaulayness fails for $k \geq 3$. Indeed, let $\mathcal{P}$ be the ordinal sum of $n$ copies of a $k$-element antichain. This poset (shown on the left of Figure 1 for $n=3$ and $k=2$ ) is $k$-Cohen-Macaulay, since removing fewer than $k$ elements from $\mathcal{P}$ yields the ordinal sum of $n$ nonempty antichains, which is Cohen-Macaulay, as an ordinal sum of Cohen-Macaulay posets, of the same rank as $\mathcal{P}$. However, $\mathcal{P}$ is not edgewise $k$-Cohen-Macaulay for $k \geq 3$ since $\mathcal{P} \ominus \mathcal{I}$ has a disconnected Hasse diagram for every rank two closed interval $\mathcal{I}$ which contains a maximal or minimal element of $\mathcal{P}$.

\section{Gorenstein* lattices}

This section proves Theorem 1.1. The following lemma provides a large class of posets $\mathcal{P}$ for which equality holds in (11) for every interval $[a, b] \subseteq \mathcal{P}$; it applies to geometric and Gorenstein* lattices (the latter have a nowhere zero Möbius function and are therefore relatively atomic, for instance, by [12, Corollary 3.9.5]).

Lemma 4.1 Let $\mathcal{L}$ be a graded, relatively atomic lattice. Then

$$
\Delta(\overline{\mathcal{L}} \ominus[a, b])=\bigcap_{a \leq x<y \leq b} \operatorname{cost}_{\Delta(\overline{\mathcal{L}})}(\{x, y\})
$$

for every closed interval $[a, b] \subseteq \overline{\mathcal{L}}$. Moreover, $\overline{\mathcal{L}} \ominus[a, b]$ is graded of the same rank as $\overline{\mathcal{L}}$.

Proof. We claim that if $u<v$ holds in $\mathcal{L}$ and at most one of $u, v$ belongs to $[a, b]$, then there exists a saturated chain in $\mathcal{L}$ with minimum element $u$ and maximum element $v$ which does not 
contain elements of $[a, b]$ other than $u, v$. The claim implies that every chain in $\overline{\mathcal{L}}$ which does not contain two elements of $[a, b]$ is also a chain in $\overline{\mathcal{L}} \ominus[a, b]$ or, equivalently, that the right-hand side of (2) is contained in the left-hand side. The reverse inclusion follows from (1). The last statement in the lemma also follows from the claim.

To prove the claim, we apply induction on the length of the closed interval $[u, v]$ of $\mathcal{L}$. Since the result is trivial if this length equals one, we may assume that $v$ does not cover $u$ in $\mathcal{L}$. By our assumption on $u, v$, we have $a \not \leq u$ or $v \not \leq b$ in $\mathcal{L}$. Suppose that $a \not \leq u$. Since $\mathcal{L}$ is relatively atomic, at least two elements of the interval $[u, v]$ cover $u$. Since the meet of these elements is $u$, at least one of them, say $w$, satisfies $a \not \leq w$. By the induction hypothesis, there exists a maximal chain of the interval $[w, v]$ of $\mathcal{L}$ which does not contain elements of $[a, b]$ other than $v$. Adding $u$ to this chain gives the desired maximal chain in $[u, v]$. A similar argument works if $v \not \leq b$.

The last two parts of the following proposition are probably known; we provide a proof for the sake of completeness. Given a simplicial complex $\Delta$, a geometric simplicial complex $\Gamma$ realizing $\Delta$ and a face $F \in \Delta$, we denote by $\left|\operatorname{st}_{\Delta}(F)\right|$ the union of the relative interiors of the geometric simplices of $\Gamma$ which correspond to the elements of $\operatorname{st}_{\Delta}(F)$. We will naturally consider $\left|\mathrm{st}_{\Delta}(F)\right|$ to be a subspace of $|\Delta|$.

Proposition 4.2 Let $\mathcal{L}$ be a Gorenstein* lattice. Then:

(i) $\Delta(\overline{\mathcal{L}} \ominus[a, b])$ is acyclic for every closed interval $[a, b] \subseteq \overline{\mathcal{L}}$ of positive rank.

(ii) $\Delta\left(\overline{\mathcal{L}} \backslash \mathcal{L}_{\geq a}\right)$ is acyclic for every $a \in \overline{\mathcal{L}}$.

(iii) $\Delta\left(\overline{\mathcal{L}} \backslash \mathcal{L}_{\leq b}\right)$ is acyclic for every $b \in \overline{\mathcal{L}}$.

Proof. To simplify notation, we set $\Delta=\Delta(\overline{\mathcal{L}})$. We first rewrite (21) as

$$
\Delta(\overline{\mathcal{L}} \ominus[a, b])=\Delta \backslash \bigcup_{a \leq x<y \leq b} \operatorname{st}_{\Delta}(\{x, y\}) .
$$

Taking geometric realizations, we get

$$
|\Delta(\overline{\mathcal{L}} \ominus[a, b])|=|\Delta| \backslash \bigcup_{a \leq x<y \leq b}\left|\mathrm{st}_{\Delta}(\{x, y\})\right| .
$$

Since $|\Delta|$ is a homology sphere, it follows from the Lefschetz duality theorem for homology manifolds [10, Theorem 70.2] and the long exact homology sequence of a pair that in order to prove (i), it suffices to show that the union in the right-hand side of (3) is contractible. For this, we will use a variant of the nerve lemma [5, Theorem 10.7]. Let $E$ be the set of two-element chains $\{x, y\}$ in the closed interval $[a, b]$ and note that $\left|\mathrm{st}_{\Delta}(F)\right|$ is a nonempty open subspace of $|\Delta|$ for every $F \in E$. Note also that, for $S \subseteq E$,

$$
\bigcap_{F \in S} \operatorname{st}_{\Delta}(F)= \begin{cases}\varnothing, & \text { if } \cup_{F \in S} F \text { is not a chain in }[a, b], \\ \operatorname{st}_{\Delta}\left(\cup_{F \in S} F\right) & \text { otherwise. }\end{cases}
$$

Moreover, $\left|\mathrm{st}_{\Delta}(G)\right|$ is a cone over any point in the relative interior of $G$, and hence contractible, for every nonempty $G \in \Delta$. Thus, by [5, Theorem 10.7], the union in the right-hand side of (3) is homotopy equivalent to the (geometric realization of the) simplicial complex on the ground set $E$ whose faces are the sets $S \subseteq E$ for which $\cup_{F \in S} F$ is a chain in $[a, b]$. Clearly, the latter 
complex is a cone with apex $\{a, b\}$ and hence contractible. Therefore, so is the union in the right-hand side of (3). This completes the proof of (i).

To verify part (ii), note that $\left|\Delta\left(\overline{\mathcal{L}} \backslash \mathcal{L}_{\geq a}\right)\right|$ is homotopy equivalent to $|\Delta| \backslash\left|\Delta\left(\mathcal{L}_{\geq a}\right)\right|$ by Lemma 2.1. Since $\Delta\left(\mathcal{L}_{\geq a}\right)$ is a cone and hence contractible, the result follows as in the proof of part (i). Part (iii) follows from part (ii) by passing to the dual lattice.

Proof of Theorem 1.1. Let $\mathcal{L}$ be a Gorenstein* lattice and $[a, b]$ be a nonempty closed interval in $\mathcal{L}$ (intervals without subscripts in this proof are meant to be intervals in $\mathcal{L}$ ). By Lemma 4.1 , the poset $\overline{\mathcal{L}} \ominus[a, b]$ is graded of the same rank as $\overline{\mathcal{L}}$.

To prove that it is Cohen-Macaulay set $\mathcal{Q}:=\mathcal{L} \ominus[a, b]$, consider elements $u, v \in \mathcal{Q}$ with $u<v$ and note that at most one of $u, v$ belongs to $[a, b]$. We need to show that the open interval $(u, v)_{\mathcal{Q}}$ has vanishing reduced homology at ranks smaller than the rank of $(u, v)_{\mathcal{Q}}$. This is clear if $[a, b]$ and $[u, v]$ have void intersection, or exactly one common element, since then $[u, v]_{\mathcal{Q}}=[u, v]_{\mathcal{L}}$ is a Gorenstein* lattice. Otherwise, we have $a \vee u<b \wedge v$, and hence $a<v$ and $u<b$, in $\mathcal{L}$. Note that we may not have $a \leq u$ and $v \leq b$ in $\mathcal{L}$. Unraveling the relevant definitions and using the claim in the proof of Lemma 4.1, we find that

$$
(u, v)_{\mathcal{Q}}= \begin{cases}(u, v) \ominus[a \vee u, b \wedge v], & \text { if } a \not \leq u \text { and } v \not \leq b, \\ (u, v) \backslash(u, b \wedge v], & \text { if } a \leq u, \\ (u, v) \backslash[a \vee u, v), & \text { if } v \leq b,\end{cases}
$$

where the equality in the last two cases follows from the fact that the elements of the half-open intervals of $\mathcal{L}$ being removed are no longer greater than $u$ (respectively, less than $v$ ) in $\mathcal{Q}$.

Since $[u, v]$ is a Gorenstein* lattice, Proposition 4.2 implies that $(u, v)_{\mathcal{Q}}$ is acyclic in each case and the proof follows.

\section{$5 \quad$ Remarks}

Shellability of simplicial complexes and posets is a combinatorial notion which is stronger than Cohen-Macaulayness; see, for instance, [5, Section 11]. A classical result of Bruggesser and Mani [7] implies that face lattices of convex polytopes are shellable. We call a poset $\mathcal{P}$ edgewise strongly shellable if $\mathcal{P} \ominus \mathcal{I}$ is shellable of the same rank as $\mathcal{P}$ for every closed interval $\mathcal{I} \subseteq \mathcal{P}$.

Question 5.1 Are the proper parts of face lattices of convex polytopes edgewise strongly shellable?

An affirmative answer has been given by the second author for Boolean and cubical lattices.

It would be interesting to find classes of edgewise strongly Cohen-Macaulay posets other than that provided by Theorem 1.1. Given the rich Cohen-Macaulay connectivity properties of geometric lattices [1] 3] [4, Section 3] [14] (see the discussion in [6, Section 3]), it seems reasonable to expect that the following question has an affirmative answer.

Question 5.2 Is the poset $\overline{\mathcal{L}}$ edgewise strongly Cohen-Macaulay for every geometric lattice $\mathcal{L}$ ?

One may even guess that every 2-Cohen-Macaulay poset which forms the proper part of a lattice is edgewise strongly Cohen-Macaulay. This statement is false, as the following example shows. Let $\Delta$ be the simplicial complex on the ground set $\{a, b, c, d, e\}$ consisting of all proper subsets of $\{a, b, c, d\}$ and $\{b, c, d, e\}$ and let $\mathcal{L}$ be the face lattice of $\Delta$, meaning the poset having 
a $\hat{0}$ and $\hat{1}$ for which $\overline{\mathcal{L}}$ is the set of nonempty faces of $\Delta$, ordered by inclusion. Then $\mathcal{L}$ is a lattice and $\overline{\mathcal{L}}$ is 2-Cohen-Macaulay, since $\Delta(\overline{\mathcal{L}})$ is the barycentric subdivision of the 2-Cohen-Macaulay complex $\Delta$. We set $u=\{b\}$ and $v=\{b, c, d\}$ and leave to the reader to check that the poset $(\overline{\mathcal{L}} \ominus[u, v])_{>u}$ is disconnected of rank one. This implies that $\overline{\mathcal{L}} \ominus[u, v]$ is not Cohen-Macaulay and hence that $\overline{\mathcal{L}}$ is not edgewise strongly Cohen-Macaulay.

The lattice of the previous example is supersolvable [12, Example 3.14.4]. Thus, the proper parts of supersolvable lattices with nowhere zero Möbius function are not always edgewise strongly Cohen-Macaulay either (these posets were shown to be doubly Cohen-Macaulay by Welker [16]). On the other hand, it is an interesting problem to determine the edgewise CohenMacaulay connectivity of noncrossing partition lattices and posets of injective words; see [2, 8] and references therein.

We close with the following question; an affirmative answer would extend a well known property of 2-Cohen-Macaulay posets to all edgewise 2-Cohen-Macaulay posets.

Question 5.3 Is it true that $\widehat{\mathcal{P}}$ has a nowhere zero Möbius function for every edgewise doubly Cohen-Macaulay poset $\mathcal{P}$ ?

\section{References}

[1] K.A. Adiprasito and A. Björner, Filtered geometric lattices and Lefschetz section theorems over the tropical semiring, preprint, 2014, 36pp, arXiv:1401.7301.

[2] C.A. Athanasiadis, T. Brady and C. Watt, Shellability of noncrossing partition lattices, Proc. Amer. Math. Soc. 135 (2007), 939-949.

[3] K. Baclawski, Cohen-Macaulay connectivity and geometric lattices, European J. Combin. 3 (1982), 293-305.

[4] A. Björner, Shellable and Cohen-Macaulay partially ordered sets, Trans. Amer. Math. Soc. 260 (1980), 159-183.

[5] A. Björner, Topological methods, in Handbook of Combinatorics (R.L. Graham, M. Grötschel and L. Lovász, eds.), North Holland, Amsterdam, 1995, pp. 1819-1872.

[6] A. Björner, Let $\Delta$ be a Cohen-Macaulay complex..., preprint, 2014, 15pp, arXiv: 1411.2165.

[7] H. Bruggesser and P. Mani, Shellable decompositions of cells and spheres, Math. Scand. 29 (1971), 197-205.

[8] M. Kallipoliti and M. Kubitzke, A poset fiber theorem for doubly Cohen-Macaulay posets and its applications, Ann. Comb. 17 (2013), 711-731.

[9] N. Matsuoka and S. Murai, Uniformly Cohen-Macaulay simplicial complexes, preprint, 2014, 15pp, arXiv:1405.7438.

[10] J.R. Munkres, Elements of Algebraic Topology, Addison-Wesley, Reading, MA, 1984. 
[11] R.P. Stanley, Combinatorics and Commutative Algebra, second edition, Birkhäuser, Basel, 1996.

[12] R.P. Stanley, Enumerative Combinatorics, vol. 1, second edition, Cambridge Studies in Advanced Mathematics 49, Cambridge University Press, Cambridge, 2011.

[13] M. Wachs, Poset Topology: Tools and Applications, in Geometric Combinatorics (E. Miller, V. Reiner and B. Sturmfels, eds.), IAS/Park City Mathematics Series 13, pp. 497-615, Amer. Math. Society, Providence, RI, 2007.

[14] M.L. Wachs and J.W. Walker, On geometric semilattices, Order 2 (1986), 367-385.

[15] J.W. Walker, Topology and Combinatorics of Ordered Sets, Ph.D Thesis, MIT, 1981.

[16] V. Welker, On the Cohen-Macaulay connectivity of supersolvable lattices and the homotopy type of posets, European J. Combin. 16 (1995), 415-426. 